\title{
Analyzing IT Governance Initiatives with Game Theory: A Systematic literature Review
}

\author{
Maximiliano M. de Faria ${ }^{1}$, Eber Assis Schmitz ${ }^{1}$, Antonio Juarez Alencar ${ }^{1 *}$, Mônica Ferreira da \\ Silva ${ }^{1}$, Petros Sotirios Stefaneas ${ }^{2}$ \\ ${ }^{1}$ The Tércio Pacitti Institute, Federal University of Rio de Janeiro, Brazil. \\ 2 Department of Mathematics, National Technical University of Athens, Greece. \\ * Corresponding author. Tel.: +55 21 3938-3224; email: antonio.juarez.alencar@gmail.com \\ Manuscript submitted April 11, 2015; accepted July 2015. \\ doi: 10.17706/jsw.10.9.1056-1069
}

\begin{abstract}
These days information technology (IT) is an integral part of all business functions. Hence, managing IT resources efficiently and effectively has become a challenging undertaking. In this context, IT governance has presented itself as a management tool that allows decision makers to focus their efforts on value creation activities that are closely aligned with business strategy. However, IT governance tends to modify the power structure within organizations. This frequently leads to conflicts, which can be analyzed with game theory. This paper presents a systematic literature review of the use of game theory to support IT governance initiatives. It is intended to be a source of information for researchers who are involved with the analysis of IT governance structures in both the private and public sectors.
\end{abstract}

Key words: Game theory, IT governance, literature review, IT strategy, IT resource management.

\section{Introduction}

Information Technology (IT) has become one of the most important drivers of economic growth at the beginning of the $21^{\text {st }}$ century [1]. Not only does it provide companies with the means to gain competitive advantage, but also to increase market and mind share [2]. The literature is full of real-world examples in which the use of IT helps to reduce time to market and improve product quality [3]. This holds true for many types of IT related projects, including business information-system evolution, decision support system construction and mobile application development. However, as there is no free lunch [4], in order to take advantage of these benefits companies must promote the adoption of concepts and methods that favor the effective use of technological resources [5].

IT governance models are arrangements containing a set of management rules, a distinct organizational structure and a collection of business processes [6]. By enabling better planning on the use of information technology resources, IT governance models help organizations to meet the challenges posed by different market scenarios [7]. Moreover, these arrangements favor the adoption of more effective and transparent management styles, which increase accountability and reduce information asymmetry within organizations [8].

It is commonly accepted that well-structured governance models are among the main influencing factors in the effective and efficient use of IT resources [9]. Also, as competition for market space increases worldwide, many companies have turned to IT as a source of innovation and competitive advantage. As a 
result, the implementation of successful business strategies is becoming highly dependent on the existence of well-conceived information-technology governance structures. All of this requires executives to be knowledgeable about the processes and activities that facilitate the implementation of IT governance [10].

In spite of the benefits that IT governance may yield, the implementation of these models tends to be full of obstacles. This stems from the existence of both internal and external factors, which are often barriers to organizational growth [11]. Conflicting needs among stakeholders, lack of financial support and resistance to change are examples of internal factor that may hinder the adoption of IT governance models. Changing consumer habits, competitors' actions and sudden changes in the political and economic scenarios are examples of external factors [12].

IT governance is full of situations that promote both competition and collaboration among interested parties [13]. It is usually assumed that everyone who is actively involved with IT governance wants the organization to succeed, favoring a collaborative effort. However, organizational changes brought in by IT governance may change the political and financial power enjoyed by different players, leading to conflict.

Over the course of time game theory has emerged as a technique that can be successfully used to analyze conflicting situations between different parties [14]. An interesting example occurs when the IT governance committee of a large organization considers making it mandatory for its software-development service providers to have an expensive hard-to-get quality assurance certificate. In these circumstances, the service providers may choose to

(a) fight the new requirement, taking the matter to a higher managerial board,

(b) bear the extra cost required to obtain and maintain the certificate,

(c) obtain the certificate and keep providing services to the organization, but at a higher price,

(d) fulfill the ongoing contracts and cease proving development services to the organization, or

(e) negotiate with the governance committee.

If service providers decide to fight the new requirement or threaten to cease providing development services, the governance committee may consider fighting back or giving up the certificate requirement. On the other hand, if service providers indicate that they are willing to bear the extra cost, it is likely that the governance committee will feel confident and will go ahead with the idea of requiring the certificate. Moreover, if the service providers decide to raise their prices, they risk losing a profitable client. Finally, there is a chance that the interested parties may resolve to negotiate and try to reach an agreement.

Note that if the players and their respective choices are well known and their actions influence one another's decisions, then a game is established [15]. Moreover, there is plenty of evidence in the literature showing that game theory can be successfully used to analyze the games that people play at the workplace [16], [17] and facilitate the implementation of corporate governance processes [18]-[20].

This paper analyses the role played by game theory in the conception, deployment and management of IT governance. This is based on a systematic and thorough review of the existing literature on the subject. It is intended to be a source of information for those who are involved with the study of IT governance in both the private and public sectors.

The remainder of this paper is organized as follows. Section 2 presents the concepts and methods used in the systematic literature review (SLR). Section 3 discusses the planning of the SLR, including the research questions that directs the systematic review. Its execution is analyzed in Section 4. Section 5 presents the results of the SLR. The conclusions of this paper may be found at Section 6 .

\section{Systematic Literature Review}

SLR is a type of literature review that provides answers to specific research questions. It follows a well-defined sequence of steps leading to the identification, analysis and synthesis of relevant information 
that helps to answer those questions [21].

SLR is an important part of research projects that seek to make significant contributions to science and technology. When properly done it provides robust up-to-date information on the subject under consideration. This information is organized to facilitate reading and understanding. Therefore, in general, its results can be immediately used by others, aiding the development of new projects [22]. The main characteristics of a SLR are summarized in Table 1.

Table 1. Main Characteristics of a SLR

\begin{tabular}{|ll|}
\hline \multicolumn{2}{|l|}{ Characteristic } \\
\hline \hline$\checkmark$ & SLR starts with the definition of a protocol outlining a research plan \\
\hline$\checkmark$ & It proceeds by showing how the plan is going to be carried out and what is intended to be reported \\
\hline$\checkmark$ & It is based on a predefined search strategy aimed at retrieving relevant scientific and technical information \\
\hline$\checkmark$ & It includes clear criteria for the selection and analysis of relevant work \\
\hline$\checkmark$ & $\begin{array}{l}\text { It is composed of well-defined and documented steps, which elevate the results of the SLR to the status of } \\
\text { robust scientific research }\end{array}$ \\
\hline
\end{tabular}

Although widely applied in medical research, SLR should not be limited to this area. As its benefits become more widespread, SLR can be expected to grow in use and become common practice in other scientific and technological areas. Psychology, sociology and education are at the forefront of the adoption of SLR. Researchers in these areas are using SLR to identify and analyze unexplored and under explored subjects, paving the way to new research and development initiatives [23].

This paper follows the ideas of Barbara Kitchenham and the Center for Review and Dissemination (CRD) of the University York on SLR [21], [23]-[25]. Table 2 outlines the steps that should be taken in order to carry out a systematic literature review.

Table 2. Steps Comprising a SLR

\begin{tabular}{|c|ll|}
\hline Step & Activity \\
\hline \hline 1 & Planning: \\
& $\checkmark$ & Identification of technological and non-technological resources required by the systematic review \\
& $\checkmark$ & Development of the review protocol, including the necessary planning for all review stages \\
\hline 2 & Executing: \\
& $\checkmark$ & Execution of the search strategy defined in the planning phase \\
& $\checkmark$ & Selection of relevant studies \\
& $\checkmark$ & Analysis of the studies identified in the previous sub-step \\
& $\checkmark$ & Evaluation of the relevance of the selected studies \\
& $\checkmark$ & Extraction and synthesis of relevant information \\
\hline 3 & Reporting: \\
& $\checkmark$ & Generation of qualitative and quantitative indicators \\
& $\checkmark$ & Reporting the results of the SLR \\
\hline
\end{tabular}

In the planning step the SLR objectives are laid down together with the repositories of scientific and technical work that are going to be consulted, the search terms to be used to select related work and the criteria used to discard non-relevant work. In the executing step the search terms are applied to the repositories described in the first step. Moreover, the results are analyzed and non-relevant works are discarded. Finally, in the reporting step the results obtained in the previous steps are organized, summarized and reported. Each of these steps are detailed in the next sections.

It should be noted that although the SLR activities outlined in Table 2 are presented as a sequence of steps, in many circumstances they are executed interactively. For example, an initial planning may be 
followed by an execution attempt, whose result presents opportunities for further planning and subsequent execution and reporting.

\section{SLR Planning}

As suggested in Section 2, this SLR starts with the development of a review protocol, which describes its objectives and the activities leading to the final reports.

\subsection{Review Objectives}

The objectives of this SLR are:

(a) Surveying recent academic publications that deal with the application of game theory to support IT governance

(b) initiatives;

(c) Identifying how IT governance initiatives can benefit from the use of game theory;

(d) Determining what IT governance models and areas can be analyzed using game theory and

(e) Establishing a basis for future scientific publications and research projects.

\subsection{Research Questions}

The second step in the review protocol is to specify the research questions to be answered by the SLR. These research questions are presented in Table 3.

Table 3. Research Questions

\begin{tabular}{|c|l|}
\hline Id & Question \\
\hline \hline $\mathrm{RQ}_{1}$ & How has game theory been used to support IT governance initiatives? \\
\hline $\mathrm{RQ}_{2}$ & What are the advantages and disadvantages of such use? \\
\hline $\mathrm{RQ}_{3}$ & Which game theory concepts, methods and techniques have been used to support IT governance? \\
\hline $\mathrm{RQ}_{4}$ & What IT governance activities have been carried out with the support of game theory? \\
\hline $\mathrm{RQ}_{5}$ & What are the opportunities for new research projects and publications? \\
\hline
\end{tabular}

It should be noted that they are closely aligned with the research objectives that have just been described. For instance, $R Q_{1}$ is aligned with SLR objective (a) and (c), and $R_{2}$ with (b). Moreover, $R_{3}$ and $R Q_{4}$ are aligned with objective (c), and $\mathrm{RQ}_{5}$ with (d).

\subsection{The Search Terms}

Given the set of research questions $\mathrm{RQ}_{1}, \ldots, \mathrm{RQ}_{5}$, it is usually straightforward to derive the search terms that should be used to consult repositories of scientific and technical works. A search term is a logical expression composed of operands and logical operators. Table 4 introduces the search terms used in this SLR.

Table 4. Search Terms

\begin{tabular}{|l|l|}
\hline \multicolumn{1}{|c|}{ Dimension } & \multicolumn{1}{c|}{ Term } \\
\hline \hline Population & "governance" OR “IT governance" \\
\hline Scope & "COBIT" OR "ITIL" OR "strategic alignment" OR "value delivery" OR "risk" \\
\hline Intervention & "game theory" OR "game theoretic" OR "gamification" OR "game concepts" \\
\hline Purpose & $\begin{array}{l}\text { "implicate" OR “implement" OR "perform" OR "evaluate" OR "estimate" OR "analyse" OR } \\
\text { "analyze" OR "approach" OR "support" }\end{array}$ \\
\hline Object & "initiative" OR "model" OR "framework" OR "activities" OR "methods" OR "techniques" \\
\hline
\end{tabular}


Based on the ideas of Barbara Kitchenham on SLR (op. cit.) a structure composed of five dimensions (i.e. population, scope, intervention, purpose and object) is used to classify the terms in Table 4 . This is expected to facilitate reading and understanding. The structure is called $\Psi \mathrm{PO}^{1}$ for obvious reasons.

In YPO terminology population refers to the knowledge areas that are relevant to the SLR. The themes of interest within the population are listed in the scope dimension. The intervention describes the paradigms, methods, techniques and concepts that may have been applied to the populations under consideration. The purpose describes the actions used to apply the items listed in intervention to those listed in population. Finally, the object indicates the objects upon which the actions may have been applied.

The search term used in SLR carried out in this paper is presented in Table 5. It is a composite of the expressions presented in Table 4. It should be noted that this composite is the result of a disjunction between the population and scope combined with conjunctions of the remaining dimensions, i.e. intervention, purpose and object.

Table 5. Composite of Search Terms

\begin{tabular}{|c|}
\hline Composite term \\
\hline 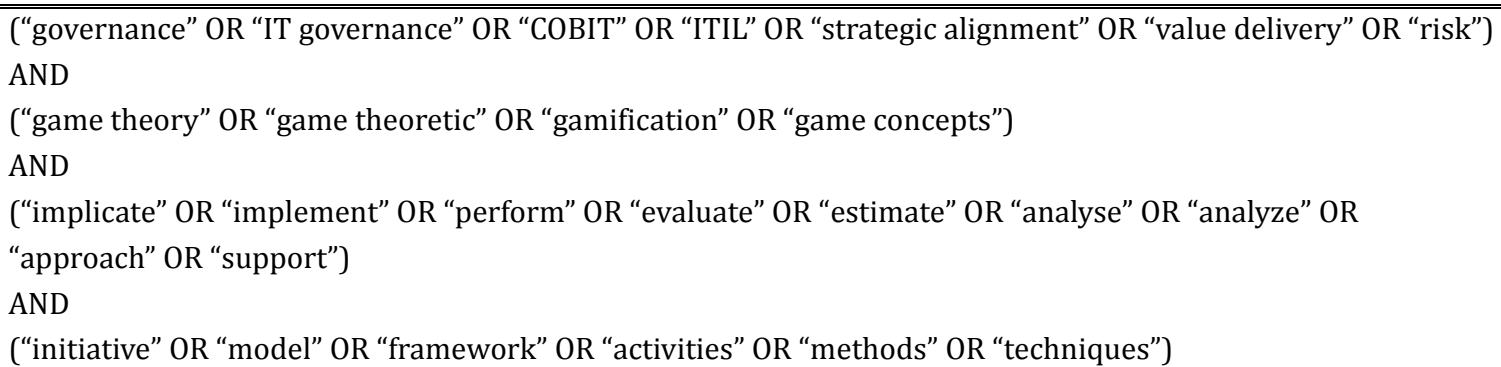 \\
\hline
\end{tabular}

\subsection{Repositories of Scientific Information}

The next step in the planning stage is to define the repositories of scientific and technical works to be used in the execution of the systematic literature review. Table 6 presents these repositories.

Table 6. Repositories of Scientific and Technical Works

\begin{tabular}{|l|l|}
\hline Repository & Internet address \\
\hline \hline DBLP Computer Science Bibliography & dblp.uni-trier.de \\
\hline CAPES periodicals & www.capes.gov.br \\
\hline Web of Science & wokinfo.com \\
\hline Scopus & www.elsevier.com/online-tools/scopus \\
\hline Google Scholar & scholar.google.com.br \\
\hline IEEE Explorer & ieeexplore.ieee.org \\
\hline
\end{tabular}

The CAPES periodicals is an Internet portal supported by the Brazilian Government. It offers access to the content of scientific and technical publications in all knowledge areas. This includes periodicals, conferences, patent records, standards, dissertations and theses.

\subsection{Search Constraints}

Since English is the standard language for scientific publications regarding the population and sub-population dimensions, the SLR reported in this paper considers only works written in that language. Among these, only papers published in journals and proceedings of conferences, congresses, symposiums

\footnotetext{
${ }^{1}$ It is pronounced as psipo.
} 
and workshops are to be selected. Moreover, these papers are required to have been published between January 2004 and January 2015.

Therefore, the SLR presented in this paper covers about ten years of academic research and technical development. Whenever possible it is established that the papers of interest are those that discuss subjects related to computer science, engineering, decision science, social sciences, business, management, economics and finance. Furthermore, paper selected from the depositories of scientific and technical information are required to satisfy the criteria established in Table 7.

Table 7. Inclusion Criteria

\begin{tabular}{|c|l|}
\hline Id & Criterion \\
\hline $\mathrm{IC}_{1}$ & The paper uses game theory to help with the implementation of governance or IT governance initiatives \\
\hline $\mathrm{IC}_{2}$ & $\begin{array}{l}\text { The paper introduces concepts, methods and techniques that can potentially be applied to governance or IT } \\
\text { governance }\end{array}$ \\
\hline $\mathrm{IC}_{3}$ & $\begin{array}{l}\text { The paper uses game theory to analyze activities, models or frameworks related to the implementation of } \\
\text { governance or IT governance }\end{array}$ \\
\hline $\mathrm{IC}_{4}$ & The paper discusses the trade-offs of applying game theory to governance or IT governance initiatives \\
\hline
\end{tabular}

These criteria are derived from the research questions presented in Table III, so as to keep paper selection aligned with the objectives of this paper. In this respect, $R_{1}$ gave rise to $I_{1}, R_{2}$ to $I C_{2}$ and so on and so forth. Papers are selected for further analysis, if they satisfy at least one of the inclusion criteria presented in Table 7.

It is important to mention that the scope of the search for relevant work has been widened at this point. The inclusion criteria presented in Table 7 allow for papers that are related to both IT and other types of governance. The reasons for this are simple. It is possible that IT governance can draw ideas from the use of game theory to support closely related initiatives.

\section{SLR Execution}

This section describes the SLR executing step in which the composite term described in Table 5 is used to select papers from the repositories presented in Table 6 . Additionally, these papers are analyzed with the aim of confirming their relevance to the SLR.

\subsection{Identification and Selection of Studies}

As a result of using the composite term as a filter for the works stored in the repositories, 583 papers were put aside for further analysis. Figure 1 shows the amount of selected papers grouped by repository of scientific and technical work. It should be noted that over half of the selected works originate from Google Scholar. The next most represented repositories are CAPES periodicals and Scopus.

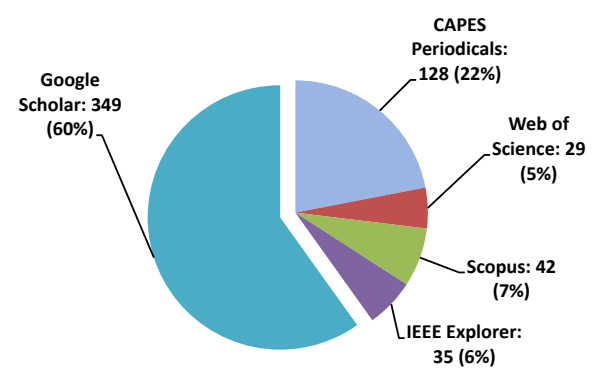

Fig. 1. Amount of selected papers grouped by repository.

Among these papers 45 have been found to be duplicated, i.e. more than one of the repositories presented 
in Table 6 refer to the same work. As a result, the duplicated references were eliminated. Next the titles, abstracts and keywords of the remaining 538, i.e. 583-45, papers were subjected to the inclusion criteria presented in Table VII. As a result of all of this, 512 papers were rejected and 26 accepted. Figure 2 summarizes this information.

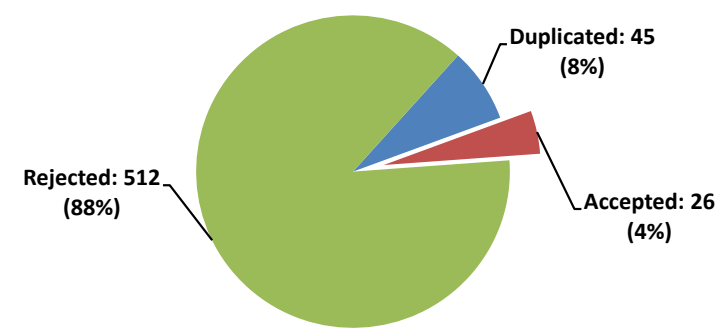

Fig. 2. The result of applying the initial selection criteria.

Finally, the full text of the remaining 26 papers, i.e. 538 - 512, were scrutinized under the criteria presented in Table 7. As a consequence, 20 papers were rejected and 6 selected. Fig. 3 summarizes this information in a pie chart. Table 8 identifies the remaining 6 papers and presents the reasons that led to their selection.

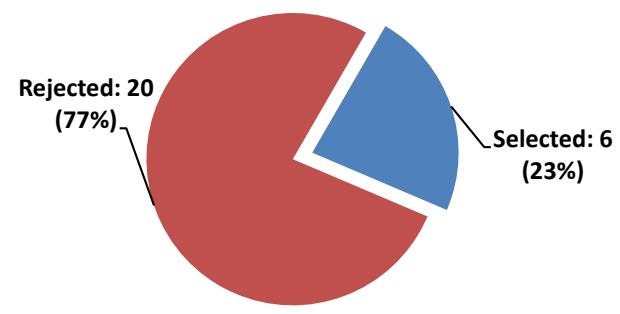

Fig. 3. Papers selected.

Table 8. Reasons for Paper Selection

\begin{tabular}{|c|c|c|c|c|c|}
\hline \multicolumn{2}{|c|}{ Paper } & \multicolumn{4}{c|}{ Inclusion Criterion } \\
\hline Id & Reference & IC $_{\mathbf{1}}$ & IC $_{\mathbf{2}}$ & IC $_{\mathbf{3}}$ & IC $_{\boldsymbol{4}}$ \\
\hline \hline Paper \#1 & {$[14]$} & & $\checkmark$ & $\checkmark$ & \\
\hline Paper \#2 & {$[26]$} & & $\checkmark$ & & \\
\hline Paper \#3 & {$[27]$} & $\checkmark$ & $\checkmark$ & $\checkmark$ & $\checkmark$ \\
\hline Paper \#4 & {$[28]$} & $\checkmark$ & $\checkmark$ & $\checkmark$ & \\
\hline Paper \#5 & {$[29]$} & $\checkmark$ & $\checkmark$ & $\checkmark$ & $\checkmark$ \\
\hline Paper \#6 & {$[30]$} & $\checkmark$ & $\checkmark$ & $\checkmark$ & $\checkmark$ \\
\hline
\end{tabular}

\subsection{Summary of the Selected Works}

What follows is a summary of the papers selected in the previous section.

\subsubsection{Paper \#1}

Abraham and Aier present a game-theory perspective on the coordination of business activities, a crucial function within corporate and IT governance [14]. The authors identify three types of games that tend to make coordination between different groups of stakeholders a challenging task, i.e. matching, battle of the sexes and assurance games.

From the analysis of these three games, they put forward a proposal to evolve the management of enterprise architecture within organizations. The proposal is twofold. The first approach proposes the use 
of active decision support activities within the realm of IT. The second promotes the same idea while moving out of the IT domain. In the latter, the decision support activities are meant to provide the necessary support for enterprise transformation.

\subsubsection{Paper \#2}

Luna, Kruchten and Moura present some preliminary ideas on how to conceive, define, and evaluate a management model for agile governance [26]. The model is expected to help organizations to attain greater agility as the same time that they pursue their business strategies. Moreover, it promotes a collaborative and adaptive organizational culture. The authors' initial insights suggest that the model should be a game played by decision makers, which uses constructs from the areas of gamification [31]-[32], fun theory [33] and game theory [18].

\subsubsection{Paper \#3}

Sun uses game theory to analyze the governance of commons [27]. In this context, commons refer to cultural and natural resources that are not owned privately, but are held in common among the members of a society.

In her work Sun advocates the adoption of a self-selecting mechanism, which favors the sustainable and effective governance of commons. Such a mechanism is shown to be superior to requiring commons to be managed according to government regulation. In addition, it performs better than allowing commons to be governed under private property rights, managed by self-organized arrangements and even overseen by self-governed bodies. Sun also claims that the self-selecting mechanism solves the problem of free riding, which happens frequently in the governance of commons.

\subsubsection{Paper \#4}

In recent years China has been the scene of some major environmental accidents. Some of them have had long lasting consequences. For example, in 2005 an explosion in a petrochemical facility in the city of Jilin caused an $80 \mathrm{~km}$ long toxic slick in the Songhua River. Over 10,000 people were displaced as a result and the water supply of millions of people in China and Russia was deeply affected [34].

Moreover, in 2007 an outbreak of toxic cyanobacteria affected the life of over two million people who lived around Lake Tai, a main source of water supply in East China. The local population was forced to stop using lake water for drinking and cooking. The stench of decay was so strong that it made breathing difficult and could be detected from over a mile away [35]. As a consequence of these and other disasters, environmental protection has become a subject of concern in China [36].

In this respect, Zhao et al. have put forward the idea of using game theory to analyze environment governance. This includes the game that government, interested companies and affected populations play in environmental crisis [28]. They claim that the insights provided by game theory can help to prevent accidents that affect the environment and increase readiness for relief actions, if the worst happens.

\subsubsection{Paper \#5}

In the realm of organizational change, Steur and Wittek claim that for the management of a transition process to be successful, it has to imply a decrease in the occurrence of severe organizational problems [29]. The difficulty in dealing with social dilemmas and unpreparedness to face disputes among employees are examples of these problems.

Steur and Wittek also claim that the number and severity of coordination problems should be expected to rise during transition periods. Note that the coordination of activities among different stakeholders is a crucial function within corporate governance. Hence, transition processes can be expected to have a considerable impact on governance.

Steur and Wittek's work allows for better planning of organizational change, as it unveils the intricacies of games that are elusively played by employees and managers in these circumstances. This favours the 
effective governance of corporate resources, including those of technological nature.

\subsubsection{Paper \#6}

A knowledge alliance (KA) in which business strategy is centered around the acquisition and sharing of knowledge [37]. In this kind of business arrangement, knowledge management and governance issues play a crucial role. Nevertheless, according to Yu, Zhang and Yang, when KAs set up a governance committee, participating parties are likely to start playing games [30]. Understanding these games and acting upon them accordingly is crucial for the success of KAs.

Consistent with this view, Yu, Zhang and Yang use game theory to analyze the games that people play in KA's governance committees and provide guidelines for management. In addition, they suggest that these committees should enhance the way knowledge is acquired and shared, so that the participating parties can perceive cost-benefit value. Also, governance committees should improve their efficiency and strengthen the efficacy of their supervising activities, as a way of keeping the KA on track.

\section{SLR Reporting}

At the outset, the SLR performed in this paper undertook to provide answers to some key research questions. See Table 3 in this respect. The answers to these questions are presented below.

\subsection{Answering RQ1: How Has Game Theory Been Used to Support IT Governance Initiatives?}

Perhaps the most striking information that can be extracted from the SLR reported in this paper is that none of the selected works deals with the governance of information technology.

This indicates a huge gap in the research work that is being carried out around the world. Not only is IT part of the business strategy of many companies, but it is also an integral part of all other business functions. Moreover, IT draws a considerable amount of investment money from an organization's annual budget [38].

Nevertheless, the six selected papers use game theory to aid the implementation of other relevant aspects of governance in both the private and public sectors. For example, the use of game theory to support the governance of common resources is approached in [27]. In addition, the prevention of environmental accidents and increasing readiness for action in the aftermath of accidents with the support of game theory and governance is discussed in [28]. Moreover, the use of game theory to improve the planning of organizational change is dealt with in [29], and the use of game theory to improve the efficacy of governance committees in knowledge alliance is studied in [30].

Initial insights provided by the selected papers indicate that game theory could be used in similar ways to aid IT governance initiatives. However, more research will have to be done in this respect.

\subsection{Answering RQ2: What Are the Advantages and Disadvantages of Such Use?}

As a whole the selected papers indicate that the games that people play can substantially affect the success of business initiatives [16-17]. For example, they may interfere with the efficacy and efficiency of different kinds of activities [30]. Also, they can increase the severity of the risks that projects and processes are exposed to [29].

Note that IT governance is a business function that relies on people to manage technological resources. Therefore, it is susceptible to the games they play and their consequences [39]. In this respect, all of the selected papers present game theory as a powerful set of concepts, methods and tools that help with the analysis of these games.

Therefore, IT governance should draw from the content of these papers and use game theory to ensure that more and better returns are provided from investments made in information technology.

On the other hand, game theory refers to concepts and methods that use mathematical modelling to 
analyze potentially conflicting situations among intelligent rational decision makers. However, IT and other business professionals may lack the necessary mathematical background to understand the intricacies of game theory. This fact alone may severely limit the usefulness of game theory to support IT governance initiatives.

\subsection{Answering RQ3: What Game Theory Concepts, Methods and Techniques Have Been Used to Support IT Governance?}

The games studied in game theory are mathematical structures composed of players, the relevant information available to each player at decision points, the actions they may take, and the payoffs for each possible outcome. The mathematical nature of game theory allows the precise identification of the best strategy for each player [15]. Therefore, if logical reasoning drives the players, they can use game theory to maximize their payoffs.

When it comes to governance, the selected papers indicate that game theory can help with the identification of the games that people are playing, the definition of the best possible strategy for each player, and the establishment of situations in which players can maximize their rewards through cooperation or competition.

All of this can benefit IT governance implementation. In this regard, game theory can help with the identification of the games that stakeholders are likely to play when managing common technological resources. Also, it can help with the analysis of the effect of possible game outcomes on business strategy. Moreover, it can provide guidelines on how management should deal with each situation. This allows for better planning of IT governance initiatives and also with the execution of implementation plans.

\subsection{Answering RQ4: What IT Governance Activities Have Been Carried out with the Support of Game Theory?}

Because none of the selected papers deal with the governance of information technology, no IT governance activities that have been carried out with the support of game theory could be identified.

Nevertheless, the selected papers give some valuable indications of activities that could benefit from the use of game theory. For instance, IT is one of the main drivers of change in a variety of markets. Therefore, as shown in [14], IT governance is likely to require the coordination of actions among stakeholders with conflicting interests. In these circumstances, matching games, battle of the sexes and assurance games can be expected to emerge as the games that people are likely to play. Moreover, the suggestions advocated by [29] could be used to reduce the difficulties in dealing with social dilemmas and unpreparedness to face competition among employees. These are common place in organizational changes enabled by IT.

As markets inevitably change over the course of time, flexibility is a powerful weapon to keep business competitive. Therefore, organizations tend to value flexibility when pursuing their business strategies. In this respect, the ideas presented in [26] could be used to help organizations to attain agility while IT governance is implemented.

In a sense IT equipment and professionals are common resources that are shared by all business functions. As a result, the approach used by [27] to analyze the governance of commons could be used to benefit the management of IT resources. Besides favoring the efficient use of resources, the approach also helps to solve the problem of free riding, which is not uncommon in IT initiatives.

In many countries, in both the public and private sector, IT is the basis for monitoring environmentally sensitive areas and also for disaster relief operations. As a consequence, the ideas put forward in [28] to analyze the games that government, interested companies and the affected population play in these circumstances could be used to manage IT resources more effectively.

Finally, as IT is at the center of knowledge management in many companies, the implementation of IT 
governance in cooperative arrangements could benefit from the ideas introduced in [30]. The way that management guidelines are constructed in that paper could allow IT governance to draw similar results, improving the effectiveness of IT resource management.

\subsection{Answering RQ5: What Are the Opportunities for the Development of New Research Projects and Publications?}

Despite the relevance of IT governance to both the private and public sectors, the SLR reported in this paper

demonstrates that very little research has been done in this respect. Therefore, the use of game theory to support IT governance is actually an open road for research and publication.

Many of the activities performed in the implementation of IT governance depend on people's decisions and actions. Assigning responsibilities to people, setting performance indicators, establishing priorities for different ideas and auditing processes are examples of activities in the realm of IT governance that are carried out by people.

The selected papers show that when people are required to perform these and other similar activities, they tend to find themselves in conflicting situations. In this case, people are likely to play a variety of games. Moreover, those papers indicate that game theory can be used to analyze the games that people play in these circumstances, and establish strategies and guidelines that maximize the outcome.

Not only are the games that people play while managing IT resources worth investigating. We should also consider the choices that are usually available to different players at the point of decision making.

Moreover, besides being relevant to the players individually, the outcomes of these games are also important for business performance in all of its dimensions. Hence, investigating the relations that exist between IT governance game outcomes and business performance is another opportunity for further research and publication.

Furthermore, in respect to IT governance many of the future findings from research carried out in the private sector will have to be reconsidered for the public sector and vice versa. As the objectives, management structures and cultures are very different from one another, it would be naive to consider that the findings drawn from one sector could be directly applied to the other.

\section{Conclusions}

This paper presents a systematic review of the literature aimed at revealing how IT governance can benefit from the use of game theory. The results of the systematic review show that so far no publication has addressed this subject directly. Therefore, connecting game theory to IT governance seems to be an open road for research and publication.

Nevertheless, some relevant research has been done on the use of game theory to support other kinds of governance. This has proved useful in clarifying how the use of game theory can support the governance of IT resources.

For example, while involved in IT governance activities, people are likely to play a variety of competitive and cooperative games. Moreover, how a game unfolds and its outcome and aftermath can be analyzed with the support of game theory. This can be used to maximize the outcome of each player and establish guidelines and policies that improve business performance.

However, game theory is essentially a mathematical subject. Therefore, the background of those involved with IT governance must be considered to ensure that the findings of future research are accessible. Note that not all business executives enjoy a high level mathematical background.

As a result, many IT governance practitioners may find it difficult to master the intricacies of game theory. Therefore, making the new findings accessible to the corporate realm may be difficult. These findings stand 
a better chance of making a difference in the real world, if they are accompanied by software that facilitates their use.

\section{References}

[1] Kettenia, E., Mamuneasa, T., \& Stengosa T. (2011). The effect of information technology and human capital on economic growth. Macroeconomic Dynamics. 15(5), pp. 595-615.

[2] Zafar F., \& Mahmood Z. (2014). Strategic management of technology and innovation. Global Journal of Management and Business Research, 13(12A), 36-43.

[3] ITGI. (2012). Board briefing on IT governance, IT governance institute. Retrieved on October 16, 2014, from

www.isaca.org/Knowledge-Center/Research/ResearchDeliverables/Pages/Board-Briefing-on-IT-Gove rnance-2nd-Edition.aspx

[4] Simmons, R. T. (2011). Beyond Politics: The Roots of Government Failure. Independent Institute.

[5] Dameri, R. P. (2010). Chapter: From IT compliance cost to IT governance benefits: An Italian business case. Management of the Interconnected World.

[6] Lunardi, G. L., Becker, J. L., Maada, A. C. G., \& Dolci, P. C. (2014). The impact of adopting IT governance on financial performance: An empirical analysis among Brazilian firms. International Journal of Accounting Information Systems, 15(1), 66-81.

[7] Smallwood, R. F. (2014). Information Governance: Concepts, Strategies, and Best Practices. Wiley.

[8] Joshia, A., Bollena, L., \& Hassinka, H. (2013). An empirical assessment of it governance transparency: Evidence from commercial banking. Information Systems Management, 30(2), 116-136.

[9] Rahman, A. A., Doina, P. P., \& Eugen, P. (2013). A survey in information systems: Integral part and a strategic partner for good corporate governance. Ovidius University Annals, Economic Sciences Series, 13(1), 395-400.

[10] Urbach, N., Buchwald, A., \& Ahlemann, F. (2013). Understanding IT governance success and its impact: Results from an interview study. Proceedings of the 21st European Conference on Information Systems (ECIS).

[11] Othman, M. F. I., Chan, T., Foo, E., Nelson, K. J., \& Timbrell, G. T. (2010). Barriers to information technology governance adoption: A preliminary empirical investigation. Proceedings of the 15th International Business Information Management Association Conference (pp. 1771-1787). Cairo, Egypt.

[12] Martins L. M., Moura A., da Cunha P. R., \& Figueiredo A. D. (2010). Selecting and ranking it governance practices for electric utilities. Proceedings of the 16th Americas Conference on Information Systems (AMCIS) (Paper 120). Lima, Peru.

[13] Prasad, A., Green, P., \& Heales, J. (2012). On IT governance structures and their effectiveness in collaborative organizational structures. International Journal of Accounting Information Systems, 13(3), 199-220.

[14] Abraham, R., \& Aier, S. (2012). Architectural coordination of transformation: Implications from game theory. Knowledge and Technologies in Innovative Information Systems.

[15] Tadelis, S. (2013). Game Theory: An Introduction. Princeton University Press.

[16] Compton, J. (2013). Games Managers Play: The Psychology of Supervising People \& Getting More with Less. Third Dimension Publishing.

[17] Delano, F., \& Shah, J. C. (2011). Games played in the supervisory relationship: The modern version. Relational Child and Youth Care Practice, 24(1), 177-185.

[18] Papayoanou, P., Charlesworth, D., \& Charlesworth, D. (2010). Game Theory for Business: A Primer in Strategic Gaming. Probabilistic Pub. 
[19] Jia, N. (2013). Competition, governance, and relationship-specific investments: Theory and implications for strategy. Strategic Management Journal, 34(13), 1551-1567.

[20] Emerson, K., Nabatchi, T., \& Balogh, S. (2012). An integrative framework for collaborative governance. Journal of Public Administration Research and Theory, 22(1), 1-29.

[21] Kitchenham, B. (2004). Procedures for Performing Systematic Reviews. Retrieved on September 1, 2014 from

http://tests-zingarelli.googlecode.com/svn-history/r336/trunk/2-Artigos-Projeto/Revisao-Sistematic a/Kitchenham-Systematic-Review-2004.pdf

[22] Gough, D., Oliver, S., \& Thomas, J. (2012). An Introduction to Systematic Reviews. SAGE Publications.

[23] CRD. (2009). Systematic Reviews. Retrieved on September 1, 2014, from www.york.ac.uk/inst/crd/pdf/Systematic Reviews.pdf. Site last visited.

[24] Kitchenham, B., Brereton, O. P., Budgen, D., Turner, M., Bailey, J., \& Linkman, S. (2009). Systematic literature reviews in software engineering a systematic literature review. Information and Software Technology, 51(7 - 15).

[25] Kitchenham, B., Brereton, P., Turner, M., Niazi, M., \& Budgen, D. (2009). The impact of limited search procedures for systematic literature reviews a participant-observer case study. Proceedings of 3rd International Symposium on Empirical Software Engineering and Measurement (pp. 336 - 345). Lake Buena Vista, FL, USA: ACM / IEEE.

[26] Luna, A., Kruchten, P., \& Moura, H. (2013). GAME: Governance for Agile Management of Enterprises. Bari.

[27] Sun, B. (2011, January 8-11). A model of self-selected governance on commons. Proceedings of the International Conference on Management Science and Industrial Engineering (pp. 1377-1379). Harbin, China.

[28] Zhao, L., Cao, Z., Yu, H., Yuan, L., \& Zhou, C. (2010, August 13-15). A game analysis of environmental governance: A case study of Baosteel group. Proceedings of the Third International Conference on Business Intelligence and Financial Engineering (pp. 238-242). Hong Kong, China.

[29] Steur, J., \& Wittek, R. (2005). The governance of transition processes in an organization: A cognitive mapping approach. Academy of Management Proceedings, 1.

[30] Yu, C., Zhang, Y., \& Yang, G. (2013). The game analysis of knowledge sharing behavior of knowledge alliance taking into the consumer. Proceedings of the International Conference on Management Science and Engineering (ICMSE) (pp. 1466-1471). Harbin, China.

[31] Dale, S. (2014). Gamification making work fun, or making fun of work? Business Information Review, 31(2), 82-90.

[32] Morford, Z. H., Witts, B. N., Killingsworth, K. J., \& Alavosius, M. P. (2014). Gamification: The intersection between behavior analysis and game design technologies. The Behavior Analyst, 37(1), $25-40$.

[33] Nordahl, D. (2012). Making Transit Fun! Island Press.

[34] Junyu, C., Jing, W., \& Xiao, H. C. (2010). Explosions, corruption and floods: How China's chemical plants became a national danger, News China.

[35] Stone, R. (2011, July issue). On lake taihu, china moves to battle massive algae blooms. Environment 360.

[36] Hea, G., Zhang, L., Lua, Y., \& Molb, A. P. (2011). Managing major chemical accidents in china: Towards effective risk information. Journal of Hazardous Materials, 186(2-3), 1489-1494.

[37] Huggins, R. \& Johnston, A. (2012). Knowledge alliances and innovation performance: An empirical perspective on the role of network resources. International Journal of Technology Management, 57(5), 245-265. 
[38] Drnevich, P. L., \& Croson, D. C. (2013). Information technology and business-level strategy: Toward an integrated theoretical perspective. MIS Quarterly, 37(2), 483-509.

[39] Huang, R., Zmud, R. W., \& Price, R. L. (2010). Influencing the effectiveness of it governance practices through steering committees and communication policies. European Journal of Information Systems, 19, 288-302.

Maximiliano M. de Faria is a Ph.D. student with the Federal University of the State of Rio de Janeiro (UNIRIO), Brazil. He holds a B.Sc. in information systems and an M.Sc. in computer science from UNIRIO. His research interests include software development methodologies, IT governance and game theory.

Eber Assis Schmitz is a professor of computer science with the Federal University of Rio de Janeiro (UFRJ). He holds a B.Sc. in electrical engineering from the Federal University of Rio Grande do Sul (UFRGS), an M.Sc. in electrical engineering from the Federal University of Rio de Janeiro and a Ph.D. in computer science and control from the Imperial College of Science, Technology and Medicine, London, England. His research interests include software development modeling tools, business process modeling and stochastic modeling.

Antonio Juarez Alencar is a researcher with the Federal University of Rio de Janeiro (UFRJ), Brazil. He received his B.Sc. in mathematics and M.Sc. in system engineering and computer science from UFRJ. He holds a D.Phil. in computer science from Oxford University, England. His research interests include economics of software engineering, IT strategy and risk analysis.

Monica Ferreira da Silva is a researcher with the Federal University of Rio de Janeiro (UFRJ), Brazil. She received her B.Sc. in mathematics and M.Sc. in system engineering and computer science from UFRJ. She holds a Ph.D. in business administration from the UFRJ's School of Business. Her research interests include the adoption of technology and IT strategy.

Petros Sotirios Stefaneas is a researcher with the Department of Mathematics of the National Technical University, Athens, Greece. At NTUA he coordinates the activities of the logic and formal methods group of the Algorithm Applications and Logic Laboratory. His research interests include logic and formal methods, philosophy of computer science and software verification. 